\title{
Das Konzept der umweltbedingten Krankheitslast
}

\section{The Environmental Burden of Disease Concept}

\section{(๑) $\circledast \Theta$}

\section{Autoren}

Myriam Tobollik', 4, Dietrich Plaß', Nadine Steckling'2, 4, Hajo Zeeb³, Dirk Wintermeyer ${ }^{1}$, Claudia Hornberg ${ }^{4}$

Institute

1 Fachgebiet Expositionsschätzung, gesundheitsbezogene Indikatoren, Umweltbundesamt, Berlin

2 Institut und Poliklinik für Arbeits-, Sozial- und Umweltmedizin, WHO Collaborating Centre for Occupational Health, Klinikum der Universität München, München

3 Abteilung Prävention und Evaluation, BIPS - Institut für Epidemiologie und Präventionsforschung, Bremen

4 Fakultät für Gesundheitswissenschaften, Universität Bielefeld, Bielefeld

\section{Schlüsselwörter}

Umweltbedingte Krankheitslast, Disability-Adjusted Life Year, DALY, Umwelt und Gesundheit, Summenmaß der Bevölkerungsgesundheit

\section{Key words}

Environmental Burden of Disease, Disability-Adjusted Life Year, DALY, environment and health, summary measure of population health

\author{
Bibliografie \\ DOI https://doi.org/10.1055/s-0043-110851 \\ Online-Publikation: 10.10.2017 \\ Gesundheitswesen 2018; 80: 154-159 \\ (c) Georg Thieme Verlag KG Stuttgart · New York \\ ISSN 0941-3790
}

\author{
Korrespondenzadresse \\ Myriam Tobollik \\ Fachgebiet Expositionsschätzung, gesundheitsbezogene \\ Indikatoren \\ Umweltbundesamt \\ Corrensplatz 1 \\ 14195 Berlin \\ myriam.tobollik@uba.de
}

\section{ZUSAMMENFASSUNG}

Ziel der Studie Umweltrisikofaktoren haben häufig eine hohe gesundheitliche Relevanz mit einem großen und z. T. nicht ausreichend genutzten Präventionspotenzial. Das Wissen über die komplexen Wirkungszusammenhänge zwischen Umwelt und Gesundheit ist für einige Umweltrisikofaktoren begrenzt.
Ein Instrument, das bestehende Evidenz nutzt, um die gesundheitlichen Auswirkungen für eine Bevölkerung oder Bevölkerungsgruppe zu quantifizieren, ist das Konzept der umweltbedingten Krankheitslast (Environmental Burden of Disease, EBD). Ziel des Beitrages ist es, das Konzept der umweltbedingten Krankheitslast vorzustellen sowie den Nutzen aber auch die Kritikpunkte zu benennen.

Methodik EBD fasst Morbiditäts- und Mortalitätsdaten in einem Summenmaß, dem Disability-Adjusted Life Year (DALY), zusammen und ermöglicht eine vergleichende Darstellung von Krankheitslasten. Hierfür werden Lebensjahre als Einheit genutzt. Mithilfe des attributablen Anteils kann ermittelt werden, welcher Anteil der Gesamtkrankheitslast auf umweltbedingte Risikofaktoren zurückzuführen ist.

Ergebnisse Das EBD-Konzept wird, trotz seiner zunehmenden Anwendung im internationalen Raum, häufig kritisch diskutiert, da die Einschränkung des mehrdimensionalen Konstrukts Gesundheit in einer Maßzahl zusammengefasst wird. Weitere Kritik bezieht sich auf die sozialen und normativen Konventionen, die das Konzept beinhaltet. Fehlende oder unzureichende Eingangsdaten können zudem die Qualität und Aussagekraft von EBD-Schätzungen limitieren.

Schlussfolgerung Es bedarf eines wissenschaftlichen Diskurses, in welchem Rahmen das Konzept in Deutschland genutzt werden kann und sollte.

\section{ABSTRACT}

Goal of the study Environmental risk factors are of great importance for public health with a considerable but often unused potential for prevention. However, knowledge about the complex associations between the environment and health effects is limited for some risk factors. A concept, which is using the existing evidence on associations between the impact of environmental factors and health effects, is the environmental burden of disease (EBD) concept. The aim of this article is to present the quantification method of the EBD concept and to discuss the advantages and its points of criticism.

Methods The EBD concept combines morbidity and mortality data in a single measure (Disability-Adjusted Life Year, DALY) to enable a comparative description of the burden of disease. Life years are used as measurement unit. The environmental share of the total DALYs is quantified by using the attributable fraction. 
Results Despite its increasing application especially in the international context, the method is still criticized, because by summarizing the complex construct of health in one single measurement unit, much important information about quality of life is lost. A further criticism refers to partly arbitrarily set social value choices. Additionally, missing or insufficient data can limit the quality and validity of EBD estimations.

Conclusion A scientific discourse is needed to decide to what extent the EBD approach can and should be used in Germany.

\section{Einleitung}

Umweltbedingte Risikofaktoren haben häufig eine hohe gesundheitliche Relevanz mit einem großen und z. T. noch nicht ausreichend genutzten Präventionspotenzial. Die Kenntnis um die gesundheitliche Bedeutung von umweltbedingten Risikofaktoren ist vielfach vorhanden, das Wissen um die komplexen Wirkungsmechanismen auf den menschlichen Organismus ist jedoch begrenzt. Der Umstand, dass meist mehrere Umweltfaktoren gleichzeitig einwirken und dass bei einzelnen Risikofaktoren zusätzlich Kombinationswirkungen auftreten können, erschwert eine exakte Bewertung der Bedeutung einzelner Umwelteinflüsse auf die Bevölkerungsgesundheit [1]. So ist der Mensch bspw. einer Vielzahl an unterschiedlichen Luftschadstoffen ausgesetzt, die z. T. gemeinsam auf den Organismus wirken, z. T. aber auch voneinander unabhängige gesundheitliche Wirkungen haben [2].

Die zunehmende Globalisierung und die damit verbundene steigende Komplexität der Herausforderungen des umweltbezogenen Gesundheitsschutzes sowie die Regulierungsaufgaben, die von der Umwelt- und Gesundheitspolitik bewältigt werden müssen, zeigen deutlich, dass in diesem Themenfeld problemorientiertes und evidenzbasiertes Fachwissen benötigt wird [3,4]. Ein wissenschaftsbasiertes Instrument, das entwickelt wurde, um den Einfluss von Umweltrisikofaktoren auf die menschliche Gesundheit quantitativ zu erfassen, ist das Konzept der umweltbedingten Krankheitslast (Environmental Burden of Disease - EBD). Dieses drückt die Mortalität und Morbidität einer Bevölkerung oder Bevölkerungsgruppe infolge von umweltbedingten Erkrankungen als Summenmaß in der Einheit verlorene gesunde Lebensjahre (Disability-Adjusted Life Years - DALYs) aus [5-7].

Ziel des vorliegenden Beitrags ist es, das EBD-Konzept vorzustellen und neben seinem Nutzen im umweltbezogenen Gesundheitsschutz auch die wesentlichen Kritikpunkte zu diskutieren.

\section{Hintergrund und Entwicklung des Konzept der Krankheitslast}

Das Konzept der Krankheitslast (Burden of Disease - BoD) wurde in den 1990er Jahren von der Weltgesundheitsorganisation (WHO) zusammen mit der Weltbank und der Harvard School of Public Health im Rahmen der ersten Global Burden of Disease (GBD)-Studie für die vergleichende Erfassung des Gesundheitszustands der Weltbevölkerung entwickelt [8]. Das übergreifende Ziel bei der Entwicklung des BoD-Konzeptes war es, auch nicht-tödlich verlaufende Krankheiten in gesundheitspolitische Debatten mit einzubeziehen und so den Einfluss von Krankheit und Tod auf die Bevölkerungen unterschiedlicher Länder oder Regionen der Welt umfassend quantitativ vergleichend darzustellen [9]. Denn aufgrund des demografischen und epidemiologischen Wandels reichen Sterbezahlen alleine nicht mehr aus, um den Gesundheitszustand darzustellen. Zusätzlich wurde nun auch die Zeit berücksichtigt, die in Krankheit verbracht wird, um so die gestiegene Bedeutung von chronischen und nicht-tödlichen Krankheiten abzubilden.

Das Konzept der Krankheitslast fasst Informationen zur Morbidität und Mortalität der jeweils betrachteten Bevölkerung in einer Maßzahl (DALY) zusammen [10]. Die Krankheitslast wird anhand der Anzahl an gesunden Lebensjahren, die durch vorzeitiges Versterben und das Leben mit einer Gesundheitseinschränkung verloren gehen, dargestellt [11]. Diese Informationen können u. a. bei Entscheidungsfindungen über Maßnahmen zur Verbesserung der Gesundheit von Bevölkerungen und zur Ressourcenverteilung in der Umwelt- und Gesundheitspolitik unterstützend eingesetzt werden [12]. Mithilfe zusammenfassender und standardisierter Analysen ermöglichen DALYs weitreichende Vergleiche in Bezug auf zeitliche Veränderungen von Krankheitslasten, verschiedene Länder und Bevölkerungsgruppen [13, 14].

Erstmalig wurden DALY-Berechnungen im Weltentwicklungsbericht der Weltbank 1993 vorgestellt [15]. Die erste GBD-Studie und die darauf folgenden Aktualisierungen präsentierten Krankheitslasten für die 192 WHO-Mitgliedstaaten [8, 16]. Zusätzlich führten einige Länder in der Folge nationale Studien durch, um durch spezifische und angepasste Berechnungen Ergebnisse zu erhalten, die besser für die Beantwortung nationaler Fragestellungen geeignet sind (u. a. [17, 18]). Für Deutschland liegen bislang keine nationalen Studien zur Bestimmung der Krankheitslast vor $[19,20]$.

Seit ihrem wegweisenden Einsatz in der ersten GBD-Studie wurde die Methodik aus verschiedenen Wissenschaftsdisziplinen heraus kritisiert und entsprechend weiterentwickelt [13, 21, 22]. Dies geschah zunächst federführend durch die WHO. Mit der GBD2010-Studie [6], hat das Institute for Health Metrics and Evaluation in Seattle, (IHME, http://www.healthdata.org/gbd) die Hauptverantwortung bei der Weiterentwicklung der Krankheitslastenmethodik und Quantifizierung der weltweiten Krankheitslast übernommen. Aktuelle Berechnungen liegen für das Jahr 2015 vor $[23,24]$.

\section{Berechnung von Disability-Adjusted Life Years}

Im Folgenden wird sowohl die in den aktuellen GBD-Studie verwendeten Berechnungsmethode des IHME als auch die zuvor verwendete Methode der WHO (GBD-1990-Studie) vorgestellt $[6,13]$. Wichtig bei der Interpretation des englischen Begriffs disability ist, dass dieser nicht mit dem deutschsprachigen Begriff der Behinderung gleichzusetzen ist. Disability drückt das Ausmaß an Gesund- 
heitseinbußen aus, das durch eine Krankheit, einen Unfall oder Risikofaktor verursacht wird und somit eine Abweichung vom optimalen Gesundheitszustand darstellt [20]. Der Fokus liegt dabei auf funktionalen Einschränkungen [25].

Die Maßeinheit DALY nutzt verlorene gesunde Lebensjahre als Maß für die Darstellung von Gesundheitsverlusten ( $\triangleright$ Abb. 1). Zur Ermittlung der Mortalitätskomponente wird die durchschnittliche statistische Lebenserwartung oder eine standardisierte Lebenserwartung als Referenzwert angenommen, und es wird berechnet, wie viele Lebensjahre in Bezug zu dieser aufgrund von vorzeitigem Versterben verloren gehen (Years of Life Lost due to premature mortality, YLL). Die Berechnung erfolgt durch die Multiplikation der Anzahl von Todesfällen, die je Altersgruppe einer betrachteten Erkrankung zuzuschreiben sind, mit der statistischen Restlebenserwartung zum Zeitpunkt des Todes.

Zur Ermittlung der Morbiditätskomponente werden die Jahre berechnet, die dadurch verloren gehen, dass Personen mit einer gesundheitlichen Beeinträchtigung leben (Years Lived with Disability, YLD) [13, 26]. Für die Berechnung gibt es 2 Ansätze: In der ursprünglichen Methode (GBD-1990-Studie) wird die Anzahl der inzidenten Krankheitsfälle mit der durchschnittlichen Dauer der Erkrankung und einem Gewichtungsfaktor für den Schweregrad der gesundheitlichen Einschränkung (Disability Weight) multipliziert [9]. Alternativ werden die prävalenten Fälle einer Erkrankung mit dem jeweiligen Disability Weight multipliziert, um die YLDs zu errechnen (GBD-2010-Studie) [22]. Disability Weights stellen den Schweregrad von Beeinträchtigungen auf einer Skala zwischen 0 (optimaler Gesundheitszustand) und 1 (Zustand vergleichbar mit dem Tod) dar [12, 27, 28] und ermöglichen so die Aufsummierung von Morbidität und Mortalität.

Darüber hinaus wurden in der ersten GBD-Studie verlorene Lebensjahre in unterschiedlichen Lebensphasen in Abhängigkeit vom Alter gewichtet: das arbeitsfähige Alter wurde am höchsten, jüngere und ältere Altersgruppen wurden geringer bewertet. Ebenso wurde eine Diskontierung zukünftiger Gesundheitsverluste (Krankheitslasten, die in der Zukunft auftreten, wurden niedriger gewichtet als aktuelle Krankheitslasten) vorgenommen [11]. Beide Parameter wurden kritisch diskutiert $[21,29,30]$ und werden in aktuellen Studien nicht mehr angewendet $[6,19,31]$.

\section{Erweiterung der Methodik zur Schätzung der umweltbedingten Krankheitslast}

Aufgrund des zunehmenden gesellschaftlichen und politischen Interesses an den Ursachen für die Krankheitslast wurde das Konzept der vergleichenden Risikoabschätzung entwickelt und eingesetzt. Mit diesem können die negativen Auswirkungen von umwelt- und lebensstilassoziierten Risikofaktoren auf die menschliche Gesundheit quantifiziert werden [32]. Für umweltbedingte Risikofaktoren entstand ein eigenständiges methodisches Konstrukt - die Environmental Burden of Disease (EBD)-Methodik [7, 14, 33]. Das EBDKonzept der WHO verbindet umweltassoziierte Risikofaktoren mit Krankheitslasten und nutzt hierbei ebenfalls DALYs als die zentrale Maßeinheit [5, 34]. Die Methode erweitert dabei den BoD-Ansatz, indem der Anteil an der Krankheitslast quantifiziert wird, der auf einen bestimmten (Umwelt-)Risikofaktor zurückgeführt werden kann (attributabler Anteil).

Ein Ziel des EBD-Konzeptes ist es, das Verständnis für die Zusammenhänge zwischen Umwelteinflüssen und den damit verbundenen gesundheitlichen Wirkungen zu vergrößern, indem das Ausmaß der umweltbedingten Risiken in einer einfach verständlichen Maßzahl quantifiziert wird. Anhand der Nutzung eines standardisierten und dadurch vergleichbaren Indikators für die umweltbedingte Krankheitslast können darüber hinaus umweltbedingte Risikofaktoren untereinander, aber auch z. B. mit lebensstilbezogenen Risikofaktoren verglichen und Prioritätensetzungen in Hinblick auf notwendige Interventionen und Regulierungen abgeleitet werden [5]. EBD-Informationen können zudem die Planung, Überprüfung und Bewertung früherer wie zukünftiger politischer Maßnahmen sowohl im Gesundheits- als auch im Umweltsektor unterstützen $[5,33,34]$.

Die Identifikation gesundheitlicher Auswirkungen von Umweltfaktoren ist der erste Schritt von EBD-Berechnungen. Im Weiteren wird abgeschätzt, wie hoch der attributable Anteil an der Krankheit ist, der durch einen Umweltrisikofaktor verursacht wird [35]. Für die Quantifizierung des attributablen Anteils wird ein Vergleichswert (counterfactual value) benötigt, der den Zustand der Exposition beschreibt, bei dem z. B. kein gesundheitliches Risiko besteht. Das kann der geringste Wert (theoretical minimum) (z. B. keine Luftverschmutzung), ein erreichbarer Wert (feasible mini-

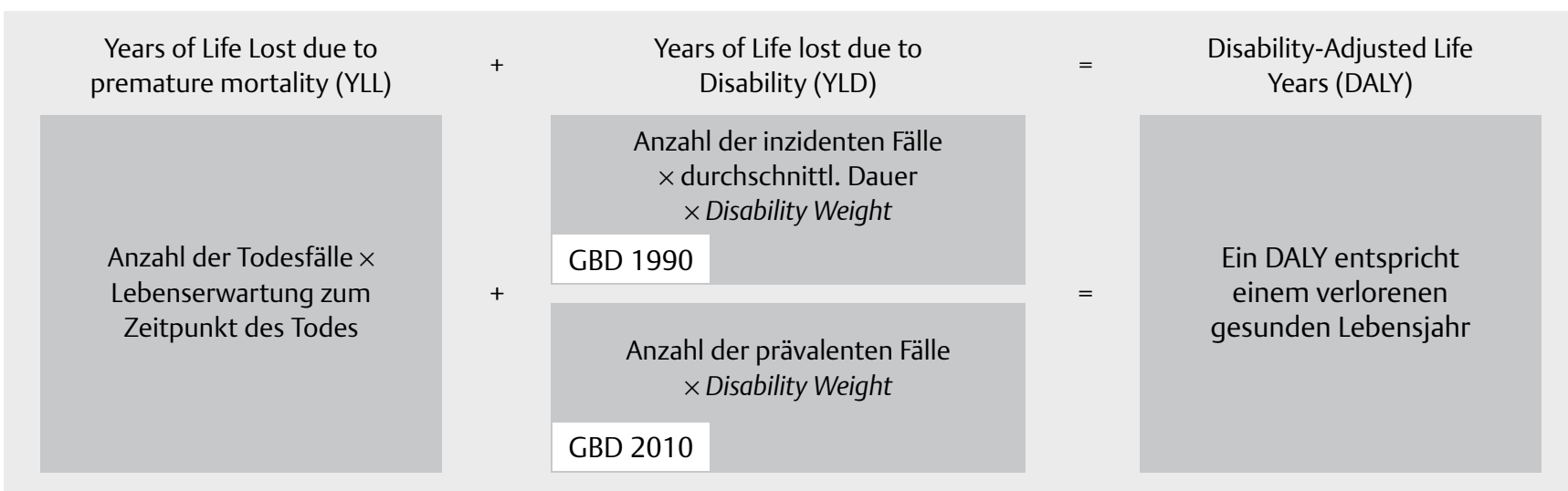

- Abb. 1 Komponenten der DALY-Berechnung. Quelle: Tobollik et al. [27], Prüss-Üstün et al. [5], Mathers et al. [41], Vos et al. [22]. [rerif] 
mum) (z. B. Verminderung der Luftverschmutzung) oder ein politisch gesetzter Grenzwert sein (limit value) [36, 37].

Zur Berechnung der EBD werden die folgenden Daten benötigt [5]:

(a) Daten zur Verteilung des Risikofaktors in der betrachteten Bevölkerung (Exposition),

(b) die Expositions-Wirkungsfunktion für den Zusammenhang zwischen Risikofaktor und den jeweils zuzuordnenden Erkrankungen,

(c) DALYs, die insgesamt - also risikofaktorunabhängig - aufgrund der betrachteten Erkrankung verursacht werden.

Die Daten zur Exposition (a) können in verschiedenen Formaten erhoben werden: z. B. aus Messungen der Schadstoffbelastung im Umweltmedium, in Humanproben oder aus Bevölkerungsbefragungen. Wenn diese Daten nicht vorhanden sind, unsicher sind oder nicht erhoben werden können, können sogenannte Szenarien entwickelt werden, in denen Annahmen über die Verteilung des Risikofaktors gemacht werden. Dabei wird die betrachtete Bevölkerung nach dem Grad der Exposition in Gruppen eingeteilt, z. B. Nichtraucher/in, ehemalige Raucher/in und Raucher/in. Hierbei ist es möglich, verschiedene Szenarien zu berechnen und so verschiedene Belastungsniveaus und deren gesundheitliche Auswirkungen darzustellen. Mithilfe solcher Szenarienberechnungen kann auch die potenzielle Reduktion der Krankheitslast infolge von gezielten Maßnahmen prognostiziert werden.

Entsprechend dem Format der verfügbaren Expositionsdaten muss eine geeignete Expositions-Wirkungsfunktion (b) ausgewählt werden. Diese Funktion beschreibt den mathematischen Zusammenhang zwischen der Exposition und den damit assoziierten Gesundheitseffekten. Solche Informationen werden mithilfe von epidemiologischen Studien erhoben, in denen das Auftreten von Erkrankungen sowohl bei Exponierten als auch bei nicht oder niedrig exponierten Personen beobachtet wird.

Mithilfe der Daten zur Exposition (a) und der Expositions-Wirkungsfunktion (b) wird der attributable Anteil anhand der in > Abb. 2 dargestellten Formel berechnet. Mit diesem werden die DALYs, die aufgrund der betrachteten Erkrankung insgesamt verloren wurden (c), multipliziert. Das Ergebnis sind die DALYs, die von dem Risikofaktor verursacht werden [5, 34].

Bspw. beträgt der attributable Anteil von Feinstaub mit einem aerodynamischen Durchmesser von bis zu 2,5 $\mu \mathrm{m}$ an Lungenkrebs für das Jahr 2009 19\% [19]. Wenn dieser Prozentsatz mit den durch vorzeitiges Versterben verlorenen Lebensjahren (649300) und den Lebensjahren, die mit Lungenkrebs verbracht wurden (8900), multipliziert wird, ergeben sich rund 125100 DALYs, die in Deutschland aufgrund der Belastung der Außenluft durch Feinstaub verloren gingen. Weitere Ausführungen zu dem Beispiel sind in diesem Erklärvideo zu finden: https://www.youtube.com/watch?v= vEe5jCGYrpl.

\section{Umgang mit Unsicherheiten}

Von zentraler Bedeutung bei Krankheitslast-Berechnungen ist die Datenqualität und Datenverfügbarkeit im Hinblick auf die benötigten Eingabeparameter, denn diese Faktoren bestimmen letztlich die Genauigkeit und Validität der Berechnung. Da jedoch die meisten Eingangsdaten Unsicherheiten beinhalten, sollte eine Unsicherheitsanalyse Teil jeder Berechnung sein [30]. Zusätzlich ist eine lückenlose Dokumentation jeden Schritts, angefangen bei der Definition der Zielsetzung bis hin zur Bewertung der Aussagekraft der Ergebnisse, notwendig. Ebenfalls sollten Eingangsdaten, die nicht explizit für den bestimmten Bezugs(zeit)raum erhoben wurden (wie bspw. Gewichtungsfaktoren oder Expositions-Wirkungsfunktionen), hinsichtlich ihrer Übertragbarkeit auf die betrachtete Bevölkerung und auf Unsicherheiten bei der Erhebung geprüft werden. Dies gilt insbesondere vor dem Hintergrund, dass Fehler in diesen ersten Arbeitsschritten zu Unsicherheiten im Gesamtergebnis führen [1]. Ebenfalls sollten Datenlücken und Einschränkungen verwendeter Eingangsdaten benannt werden. Neben dieser transparenten Dokumentation sollen zudem alle getroffenen Annahmen (z. B. verwendete Disability Weights, Diskontierung) explizit genannt werden.

\section{Kritik am Konzept der (umweltbedingten) Krankheitslast}

Ein grundsätzlicher Einwand gegen das Krankheitslastenkonzept ist, dass in diesem das eigentlich aus vielen unterschiedlichen Dimensionen und Facetten bestehende Konstrukt Gesundheit bzw. Einschränkungen dieser auf eine eindimensionale Maßzahl reduziert wird. Die Zusammenfassung von komplexen Informationen über den Verlust von Lebenszeit und Lebensqualität in eine Maß-

\section{Daten zur Verteilung \\ des Risikofaktors in der Beölkerung \\ Wirkungsfunktion}

$$
A F=\frac{\sum P_{i} R R_{i}-1}{\sum P_{i} R R_{i}}
$$

$\mathrm{AF}=$ Attributable Fraction

$\mathrm{P}_{\mathrm{i}}=$ Anteil der exponierten Bevölkerung

$\mathrm{RR}_{\mathrm{i}}=$ Relatives Risiko oder Expositions-Wirkungsfunktion

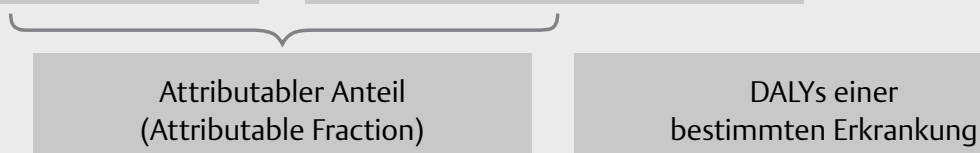

DALYs, die auf den Stressor zurückzuführen sind

Abb. 2 Methode der EBD-Berechnung [5]. 
zahl stellt eine Vereinfachung der Wirklichkeit dar, bei der wichtige Informationen, wie bspw. soziale Präferenzen, verloren gehen können [38, 39]. Insbesondere die Disability Weights werden kritisch gesehen, denn mit diesen würden Menschen aufgrund ihrer funktionalen Leistung bewertet [38]. Wichtig ist hierbei festzuhalten, dass die Disability Weights sich nicht auf ein konkretes erkranktes Individuum beziehen, sondern lediglich den durchschnittlichen Schweregrad eines Gesundheitszustands darstellen sollen. Für eine ausführliche Diskussion zu den Disability Weights siehe Nord [40] und Tobollik et al. [27].

Weitere Kritikpunkte beziehen sich auf die Annahmen zur gesellschaftlichen Bewertung des Wertes eines Lebensjahres (Diskontierung zukünftiger Gesundheitsgewinne und Altersgewichtung) und wer diese festlegen soll.

Die Wahl der Lebenserwartung (standardisierte oder berechnete Lebenserwartung basierend auf beobachteten Daten, geschlechterunterschiedliche Lebenserwartungen) beeinflusst ebenfalls die Ergebnisse, da diese als Referenz für die Berechnung der YLLs dient [26]. Je höher die gewählte Lebenserwartung, desto höher ist auch die Anzahl an YLLs.

Eine weitere Einschränkung ist die begrenzte Verfügbarkeit von Eingangsdaten. Aufgrund von lückenhaften, mangelhaften oder fehlenden Daten kann es sowohl zu einer Unterschätzung als auch zu einer Überschätzung der tatsächlichen Krankheitslast kommen. Der Mangel an qualitativ hochwertigen, repräsentativen Daten führt meist zu einer Reduktion der Komplexität von EBD-Modellen. Oftmals können Kombinationswirkungen, Komorbiditäten und Krankheitsabfolgen nicht oder nicht ausreichend berücksichtigt werden [19, 39]. Fehlen notwendige Eingangsdaten, ist es zudem oft nur möglich, einen Teil der gesamten Krankheitslast quantitativ darzustellen, was wiederum die Vergleichbarkeit von EBD-Berechnungen für verschiedene Umweltrisikofaktoren und die Ableitung von Prioritäten einschränkt. Denn eine Priorisierung alleine auf der Grundlage von DALY-Angaben, aufbauend auf dem derzeitigen Wissenstand, würde gut untersuchten umweltbedingten Risikofaktoren eine wesentlich höhere Krankheitslast zuweisen als weniger gut untersuchten - schon aus dem Grund, dass gut untersuchten Risikofaktoren zumeist viele Gesundheitsendpunkte zugeschrieben werden können. Dies lässt aber nicht unbedingt direkte Rückschlüsse auf die gesundheitliche Relevanz des umweltbedingten Risikofaktors zu [19]. Daher sollte neben der Darstellung in DALYs immer auch eine ausführliche qualitative Beschreibung des umweltbedingten Risikofaktors, seiner gesundheitlichen Wirkungen und des aktuellen Forschungsstandes erfolgen.

\section{Fazit}

Zur Berechnung der umweltbedingten Krankheitslast werden viele verschiedene Daten benötigt, die in Deutschland derzeit nur begrenzt verfügbar sind. Die Zusammenführung von Daten mithilfe der EBD-Methode kann aufzeigen, welche Datenlücken bestehen, um den Gesundheitszustand einer Bevölkerung oder Bevölkerungsgruppe umfassend darstellen zu können. Wenn ausreichende Daten vorhanden sind, kann mithilfe der DALYs aufgezeigt werden, welche Risikofaktoren die größte Krankheitslast verursachen und welche Bevölkerungsgruppen am stärksten betroffen sind. Mithilfe der DALYs können in begrenztem Maße u. a. Vergleiche zwischen ver- schiedenen Risikofaktoren und Rangfolgen erstellt werden. Diese Informationen können auch in Deutschland die Planung, Überprüfung und Bewertung von politischen Maßnahmen sowohl im Gesundheits- als auch im Umweltsektor unterstützen. Um EBD-Daten für diese Zwecke nutzen zu können, muss neben der Datenverfügbarkeit auch die Akzeptanz des Konzepts und der darin enthaltenen Konventionen verbessert werden. Es bedarf somit eines fachwissenschaftlichen Diskurses über das Konzept und dessen Anwendung in Deutschland. Hierzu ist auch die Einbeziehung der Bereiche Forschungsförderung sowie der Wissenschafts-, Umwelt- und Gesundheitspolitik erforderlich, da die Erhebung fehlender Daten bspw. durch repräsentative epidemiologische Studien mit hohen Kosten verbunden ist. Nur durch eine verbesserte Datenlage können die Ergebnisse von EBD-Berechnungen qualitativ aufgewertet, vervollständigt und damit belastbarer werden - nicht zuletzt für politische Entscheidungen im Themenfeld Umwelt und Gesundheit. Dieses gerade auch angesichts dringender Zukunftsprobleme wie Klimawandel und Schadstoffemissionen bedeutsame Themenfeld könnte mithilfe von EBD-Quantifizierungen gestärkt werden, indem die Bedeutung von Umweltfaktoren für die Gesundheit klar verständlich aufgezeigt und quantitativ dargestellt wird.

Interessenkonflikt

Die Autoren geben an, dass kein Interessenkonflikt besteht.

\section{Literatur}

[1] Briggs D. Environmental pollution and the global burden of disease. British Medical Bulletin 2003; 68: 1-24

[2] European Commission. Opinion on risk assessment on indoor air quality. Brussels: European Commission, Scientific Committee on Health and Environmental Risks (SCHER); 2007

[3] Nowotny H. Experten, Expertise und imaginierte Laien. In: Bogner A, Togersen H., Hrsg. Wozu Experten? Ambivalenzen der Beziehung von Wissenschaft und Politik. Wiesbaden: VS Verlag für Sozialwissenschaften; 2005: 33-44

[4] Knol AB, Briggs D], Lebret E. Assessment of complex environmental health problems: framing the structures and structuring the frameworks. The Science of the total environment 2010; 408: 2785-2794

[5] Prüss-Üstün A, Mathers C, Campbell-Lendrum D et al. Introduction and methods. Assessing the environmental burden of disease at national and local levels. Geneva: World Health Organization; 2003

[6] Murray C], Ezzati M, Flaxman AD et al. GBD 2010: design, definitions, and metrics. Lancet 2012; 380: 2063-2066

[7] Gao T, Wang XC, Chen R et al. Disability adjusted life year (DALY): A useful tool for quantitative assessment of environmental pollution. The Science of the total environment 2014; 511C: 268-287

[8] Murray CJL, Lopez AD. The global burden of disease: a comprehensive assessment of mortality and disability from diseases, injuries, and risk factors in 1990 and projected to 2020. Cambridge: Harvard School of Public Health on behalf of the World Health Organization and the World Bank; 1996

[9] Murray CJL, Salomon JA, Mathers CD et al. Summary measures of population health: concepts, ethics, measurement and applications. Geneva: World Health Organization; 2002 
[10] Field M], Gold MR. Summarizing population health: Directions for the development and application of population metrics. Washington DC: National Academy of Sciences; 1998

[11] Murray C]. Quantifying the burden of disease: The technical basis for disability-adjusted life years. Bull World Health Organ 1994; 72 : 429-445

[12] Mathers C, Vos T, Lopez A et al. National burden of disease studies: A practical guide. Geneva: World Health Organization (WHO); 2001

[13] Murray CJL, Salomon JA, Mathers CD. A critical examination of summary measures of population health. In: Murray CJL, Salomon JA, Mathers CD et al., (Hrsg.). Summary measures of population health; concepts, ethics, measurements and applications. Geneva: World Health Organization; 2002: 13-40

[14] McMichael A, Pastides H, Prüss A et al. Update on World Health Organization's initiative to assess environmental burden of disease. Epidemiology 2001; 12: 277-279

[15] World Bank. World Development Report 1993. Oxford: World Bank; 1993

[16] WHO. The global burden of disease: 2004 update. Geneva: World Health Organization; 2008

[17] Begg SJ, Vos ET, Barker B et al. The burden of disease and injury in Australia 2003. Canberra: Australian Institute of Health and Welfare; 2007

[18] Lai T, Habicht J, Kiivet RA. Measuring burden of disease in Estonia to support public health policy. Eur J Public Health 2009; 19: 541-547

[19] Hornberg C, Claßen T, Steckling N et al. Abschlussbericht des Projektes "Verteilungsbasierte Analyse gesundheitlicher Auswirkungen von Umwelt-Stressoren". Dessau-Roßlau: Umweltbundesamt (UBA); 2013

[20] Plass D, Vos T, Hornberg C et al. Entwicklung der Krankheitslast in Deutschland: Ergebnisse, Potenziale und Grenzen der Global Burden of Disease-Studie. Dtsch Arztebl International 2014; 111: 629-638

[21] Anand S, Hanson K. Disability-adjusted life years: A critical review. Journal of Health Economics 1997; 16: 685-702

[22] Vos T, Flaxman AD, Naghavi M et al. Years lived with disability (YLDs) for 1160 sequelae of 289 diseases and injuries 1990-2010: a systematic analysis for the Global Burden of Disease Study 2010. Lancet 2012; 380: 2163-2196

[23] Kassebaum N], Arora M, Barber RM et al. Global, regional, and national disability-adjusted life-years (DALYs) for 315 diseases and injuries and healthy life expectancy (HALE), 1990-2015: a systematic analysis for the Global Burden of Disease Study 2015. The Lancet 2016; 388: 1603-1658

[24] Forouzanfar MH, Afshin A, Alexander LT et al. Global, regional, and national comparative risk assessment of 79 behavioural, environmental and occupational, and metabolic risks or clusters of risks, 1990-2015: a systematic analysis for the Global Burden of Disease Study 2015. The Lancet 2016; 388: 1659-1724

[25] Salomon JA, Haagsma JA, Davis A et al. Disability weights for the Global Burden of Disease 2013 study. The Lancet Global health 2015; 3: e712-e723
[26] Devleesschauwer B, Havelaar AH, Maertens de Noordhout C et al. DALY calculation in practice: a stepwise approach. Int J Public Health 2014; 59: 571-574

[27] Tobollik M, Terschüren C, Steckling $N$ et al. Disability Weight - ein umstrittenes Maß zur Bewertung von Gesundheitsbeeinträchtigungen. In: László Kovács RK Ralf Lutz, (Hrsg.). Lebensqualität in der Medizin. Wiesbaden: VS Verlag für Sozialwissenschaften; 2016: 237-257

[28] Stouthard MEA, Essink-Bot M-L, Bonsel G] et al. Disability weights for diseases in the Netherlands. Rotterdam: Department of Public Health, Erasmus University Rotterdam; 1997

[29] Reidpath DD, Allotey PA, Kouame A et al. Measuring health in a vacuum: examining the disability weight of the DALY. Health Policy Plan 2003; 18: 351-356

[30] Knol AB, Petersen AC, van der Sluijs JP et al. Dealing with uncertainties in environmental burden of disease assessment. Environmental Health 2009; 8: 1-13

[31] Prüss-Ustün A, Wolf J, Corvalán C et al. Preventing disease through healthy environments: a global assessment of the burden of disease from environmental risks. Geneva: World Health Organization; 2016

[32] Mathers C, Ezzati M, Lopez A et al. Causal decomposition of summary measures of population health. In: Murray C, Salomon J, Mathers C et al., (Hrsg.). Summary measures of population health Concepts, ethics, measurement and applications. Geneva: World Health Organization; 2002: 273-290

[33] Kay D, Prüss A, Corvalan C. Methodology for assessment of Environmental burden of disease. Geneva: World Health Organization; 2000

[34] Malsch AKF, Pinheiro P, Krämer A et al. Zur Bestimmun von "Environmental / Burden of Disease" (BoD/ EBD) in Deutschland. Materialien "Umwelt und Gesundheit". Bielefeld: Landesinstitut für den Öffentlichen Gesundheitsdienst (lögd) NRW; 2006

[35] Smith KR, Corvalan CF, Kjellstrom T. How much global ill health is attributable to environmental factors? Epidemiology 1999; 10: 573-584

[36] Murray C], Lopez AD. On the comparable quantification of health risks: lessons from the Global Burden of Disease Study. Epidemiology 1999; 10: 594-605

[37] Lim SS, Vos T, Flaxman AD et al. A comparative risk assessment of burden of disease and injury attributable to 67 risk factors and risk factor clusters in 21 regions, 1990-2010: a systematic analysis for the Global Burden of Disease Study 2010. Lancet 2012; 380: 2224-2260

[38] Arnesen T, Nord E. The value of DALY life: Problems with ethics and validity of disability adjusted life years. BMJ 1999; 319: 1423-1425

[39] Sayers BM, Fliedner TM. The critique of DALYs: a counter-reply. Bulletin of the World Health Organization 1997; 75: 383-384

[40] Nord E. Disability weights in the Global Burden of Disease 2010: unclear meaning and overstatement of international agreement. Health Policy 2013; 111: 99-104

[41] Mathers CD, Bernard C, Iburg KM et al. Global burden of disease in 2002: data sources, methods and results. Global Programme on Evidence for Health Policy Discussion Paper. Geneva: World Health Organization (WHO); 2004 